\title{
Fazit und Ausblick
}

Retouren sind und bleiben ein Bestandteil der Geschäftsmodelle im OnlineHandel. Mit stetig weiter wachsendem Online-Umsatz wird auch die Zahl der Retouren zunehmen. Die Unternehmen müssen zwischen Kunden- und Kostenorientierung abwägen. Der Online-Handel profitiert einerseits vom kundenfreundlichen Widerrufsrecht, andererseits sind hohe Retourenquoten ein Ärgernis und belasten die Werthaltigkeit des Geschäftsmodells. Die Ableitung und Umsetzung einer eher kulanten oder eher restriktiven Retourenstrategie muss mit der Erhebung und Analyse der Retourengründe beginnen. Mit Maßnahmen des präventiven Retourenmanagements kann das Retourenaufkommen beeinflusst werden. Durch die dauerhafte und nachhaltige Verringerung der Retourenquote wird der Erlös optimiert, wenn es dem Online-Händler gelingt, durch präventive Maßnahmen die Kundenbindung zu erhalten und den Wertbeitrag pro Kunde zu steigern. Mit Maßnahmen des reaktiven Retourenmanagements lassen sich die durchschnittlichen Kosten pro Retoure senken. Mit einer schnellen Bereitstellung der Retouren für den erneuten Abverkauf werden die Warenverfügbarkeit und die Chance auf einen erneuten Abverkauf gesichert.

Retouren müssen also nicht als gegeben hingenommen werden. Das Kundenverhalten kann bereits vor Abschluss der Bestellung beeinflusst werden, indem die Auswahl des richtigen Produktes so einfach wie möglich gestaltet wird. Aus den in diesem essential und in der weiterführenden Literatur aufgezeigten Ansatzpunkten können Online-Händler Anregungen für ein zielgerichtetes Retourenmanagement ableiten. Die Gewichtung und Ausgestaltung der Strategien und Maßnahmen bleibt eine unternehmensindividuelle Aufgabe. Denn es wird auch in Zukunft weder in Theorie noch in der Praxis eine Patentlösung geben, welche sich für alle Online-Händler gleichermaßen umsetzen lässt. 